archives

of thermodynamics

Vol. 38(2017), No. 1, 3-26

DOI: $10.1515 /$ aoter-2017-0001

\title{
Coordination number for random distribution of parallel fibres
}

\author{
PIOTR DARNOWSKI* \\ PIOTR FURMAŃSKI \\ ROMAN DOMANSKI
}

Warsaw University of Technology, Institute of Heat Engineering, Nowowiejska 21/25, 00-665 Warsaw, Poland

\begin{abstract}
This paper presents the results of computer simulations carried out to determine coordination numbers for a system of parallel cylindrical fibres distributed at random in a circular matrix according to twodimensional pattern created by random sequential addition scheme. Two different methods to calculate coordination number were utilized and compared. The first method was based on integration of pair distribution function. The second method was the modified sequential analysis. The calculations following from ensemble average approach revealed that these two methods give very close results for the same neighbourhood area irrespective of the wide range of radii used for calculation.
\end{abstract}

Keywords: Coordination number; Random sequential addition; Parallel fibres; Fibrereinforced composite

\section{Nomenclature}

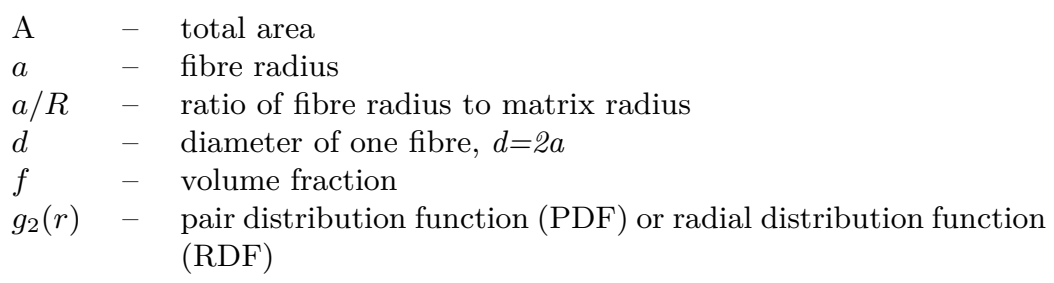

${ }^{*}$ Corresponding Author. E-mail: piotr.darnowski@itc.pw.edu.pl 


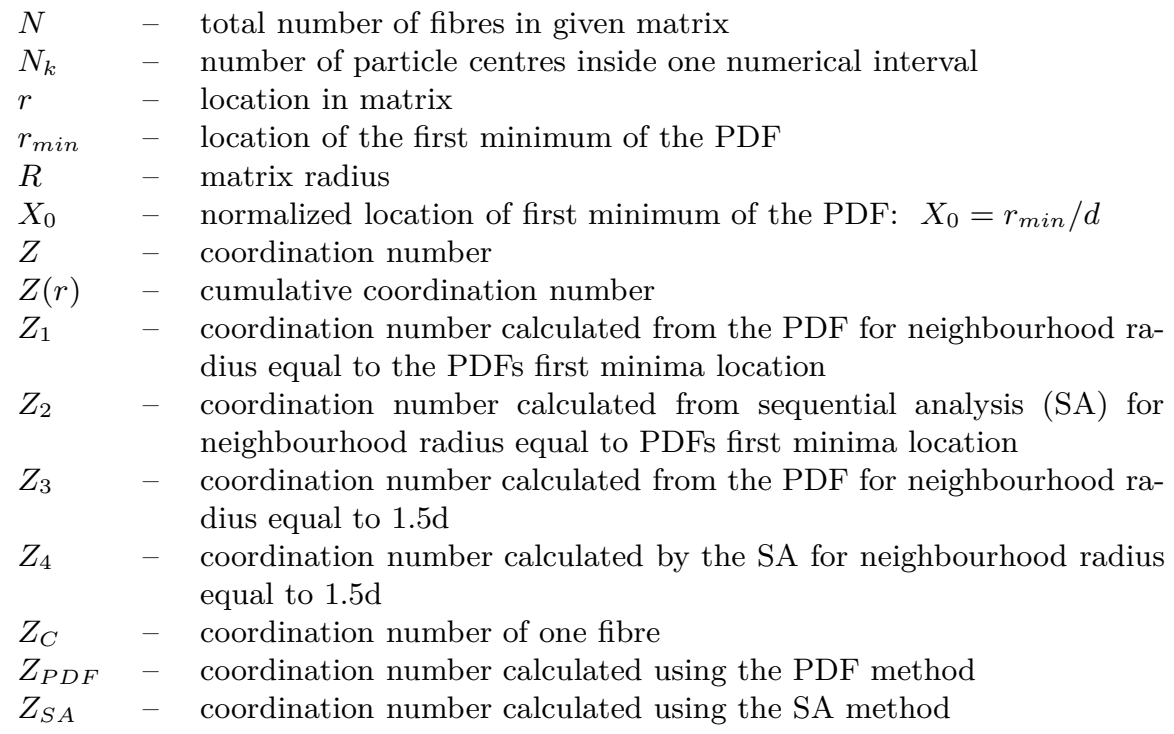

Greek symbols

$\lambda-\quad$ neighbourhood region radius normalized by fibre diameter

$\Delta r \quad-\quad$ width of one numerical interval (bin)

$\rho \quad-\quad$ particle area density (number of particles per unit area)

\section{Introduction}

Effective transport properties of heterogeneous media such as thermal conductivity, elastic moduli or dielectric or magnetic permittivity depend on properties of individual constituents, their interaction and way of distribution in the medium. One of the indicators describing microgeometry of heterogeneous media with dispersed constituents such as granular media or fibrous composites is the coordination number. It is understood as the mean number of particles which are the closest to the selected particle. Proper estimation of the coordination number may therefore allow for better tailoring of the effective properties of these materials to their applications.

The main motivation of this paper was to compare two distinctly different methods of calculating the coordination number for a composite reinforced with unidirectional aligned cylindrical fibres of constant diameter randomly distributed in a matrix. The first method is derived from the statistical mechanics and is based on integration of the pair distribution function (PDF). The second one, the sequential analysis (SA) has its origin 
in methods relevant to granular media and is based on simple counting of contacts between fibres. In the beginning ensemble averaged PDFs and CNs for thousands of geometries were found. Subsequently in the same manner, PDFs and CNs for six example cases of one geometry realization were calculated and compared with ensemble averaged results for both PDF and SA methods. The SA is applicable to any geometry, however, for the PDF method some limitations exist associated with the ability to find the pair distribution function for systems with a small number of fibres. This work should also help to answer if it is possible to assess reasonable evaluation of CNs by PDF method from a single geometry configuration of fibres and in fact it was the second motivation of this work. The third objective was to examine qualitative differences between pair distribution function of the finite geometry with radial distribution function of the infinite medium. In consequence, such examination will allow to estimate influence of geometrical boundary on CNs calculated by PDF method. An important problem associated with evaluation of CNs for geometries with random sequential addition (RSA) packing scheme is how to select the area of neighbourhood. Two approaches were here compared and applied to both CN finding methods. Additionally, the cumulative coordination numbers were calculated to give more general conclusions about that problem and to find a relation between the $\mathrm{CN}$ and the volume fraction of fibres. To authors knowledge no correlation between the mean coordination number and volume fraction for the RSA packing scheme has been presented in literature till now.

\subsection{Coordination number}

There is no strict definition of the coordination number $(\mathrm{CN})$ and it depends on the field of research considered. In chemistry the $\mathrm{CN}$ is the number of atoms directly linked by chemical bonds with the specified atom [1]. This term is also used in crystallography where it is understood as the number of atoms touching the given atom in a crystal lattice [2]. In granular media, powder technology and pebble bed theory the mean coordination number is defined as the average number of particles in direct contact with the analysed particle. In this case coordination number has a huge impact on properties like conductivity or elasticity of such materials in diverse conditions [3-6]. The statistical mechanics attempts to describe microscopically complex processes where many particles interact in a sophisticated way. Hence, there is another and more abstract way of understanding the coordination number. It is calculated as an integral of the specified function 
called the pair distribution function in given integration limits [6-8]. Such integral may give valuable information about many processes, for instance about fluid-solid phase transition [9]. The coordination number is strongly coupled with behaviour of the PDF function. Nevertheless, in the statistical approach there is no constraint on taking into account only direct contacts between particles.

A vast number of studies were conducted to describe the structure of random patterns in the granular media for both constant and variable size of particles including many different packing schemes. Special concern was given to investigate packing of spherical grains with much theoretical and experimental research referring to the coordination number as the important quantity $[10,11,5,12]$. However much less publications considered two-dimensional systems and therefore this problem became recently more interesting. Until now most engineering applications related to three dimensions, however, with the development of nanotechnology and thin layer science, two-dimensional structures become more significant. For example it was suggested that hard disk model proposed to find the CN can be useful in description of interaction of thin layers with radiation [13]. Similarly, this approach may be appropriate to describe some phenomena occurring during clustering or deposition of molecules on surfaces and description of other processes like adsorption or catalysis [14,15].

The approach presented in this paper is the combination of methods of statistical mechanics (PDF function) and a method created for granular media (SA). Coordination numbers are predicted without the necessity for fibres to be in the direct contact. Therefore a problem appears how to define a distance for which one particle is considered as the neighbour of another particle. Statistical nature of the RSA causes that the probability of perfect contact between two particles goes to zero [9]. In effect typical coordination number calculation with direct contact condition is rather useless and to omit this problem specified neighbour radius is defined $[9,14,8]$. It is worth here to mention that the sequential analysis was initially introduced for particles in direct contact, and it is extended to more general case in this paper. 


\section{Methods and geometry}

\section{$2.1 \quad$ Geometry}

The simulations were performed for two dimensional random suspensions of fibres with constant diameter distributed inside circular matrix with the fibre volume fractions corresponding to $30 \%, 40 \%$, and $49 \%$. The RSA saturation limit, i.e., the maximum possible volume fraction was estimated to be about $54 \%$. This result agrees well with the results for the so called 'hard disks jamming limit' presented in $[4,16,17]$. Simulations for fibre volume fractions exceeding $49 \%$ took too much computational time to collect satisfactory amount of data and that is the reason why the lower values were chosen. Smaller volume fractions, i.e., $40 \%$ and $30 \%$ were arbitrarily selected for comparison. A few different radii of fibres normalized to radius of matrix were considered: $a / R=0.03,0.04,0.05$, and 0.06 with $R$ being the matrix radius. These radii with the volume fractions listed above provide reasonable number of particles ranging from 83 to 544 . This range is wide enough to show some features of the coordination numbers and pair correlation functions using PDF method. It is worth here to mention that the sequential analysis can be applied in any range so there is no constraint on the number of particles used to obtain reasonable results.

\section{$2.2 \quad$ Numerical scheme}

Geometrical input to the numerical code was the volume fraction and the first guess of the fibre radius. The code started with initial values of fibre radius and area. Subsequently product of total system area and volume fraction was divided by area of one fibre to find the required number of fibres. If the expected amount of fibres inside matrix was not an integer the iteration process started. New number of fibres was set to be equal to closest integer number and the current fibre radius was changed by small steps until the volume fraction was equal to the assumed value.

A random number generator with constant probability density was used to obtain a series of random distributions of fibres inside the matrix. The following limits for the sampling process were assumed: fibres cannot intersect the outer boundary of matrix and they cannot intersect the boundary of other particles. The random sequential addition method was used to create proper distribution of fibres [4]. In this method, fibres are added one by one to the matrix and if a sampled particle violates any boundary, the 
current sampling cycle is stopped and a new particle is generated. Such procedure is repeated until the assumed amount of particles is achieved $[4,8,14]$.

After the sampling process is over the pair distribution functions (PDF) for every realization are found and the coordination numbers are calculated. The steps described above constitute the inner computation process which is conducted many times for all the analysed realizations of fibres distributions and creates the main computational loop. At the end of main computational loop the ensemble averages for all coordination numbers with standard deviations are calculated and averaged PDF is found. The computer code has been written using Alglib numerical library [17]. This library was subsequently used to find penalized regression spline to fit the PDF. One thousand realizations for analysed geometries were generated. For every realization, the first minimum of the pair distribution function was determined.

\subsection{Pair distribution function method}

The pair distribution function (PDF) or pair correlation function, $g_{2}(r)$, is one of the simplest statistical descriptors of random ensemble of particles $[18,19]$. It gives the probability of finding a particle in vicinity of other particles. In the case of isotropic system when its geometric properties are not dependent on direction and for the infinite number of particles, the PDF becomes the well-known radial distribution function (RDF). The RDF quickly tends to one for increasing distance between particles and it is significantly varying only near the particle-particle contact region. The radial distribution function (Figs. 1-3) has at least one peak, which lies in the point of contact $r / d=1.0$, where $d$ is a particle diameter. In the case of nonoverlapping fibres, the pair distribution function or radial distribution function is equal to zero for $r / d<1.0$.

The coordination number and the more general cumulative coordination number for the analysed structures was found by integration of the PDF function. The cumulative coordination number for two dimensions is defined as $[14,8]$

$$
Z(r)=\int_{0}^{r} 2 \pi r g_{2}(r) \rho d r .
$$

The mean coordination number

$$
Z_{P D F}=\int_{0}^{\lambda d} 2 \pi r g_{2}(r) \rho d r .
$$


is the value of the cumulative coordination number for upper integration limit corresponding to $r / d=\lambda$, where $\lambda$ is a radius of neighbourhood. Usually mean coordination number is calculated for direct contact of particles, i.e., $\lambda=1.0$ [4]. However with the RSA method used for the nonoverlapping fibres the cumulative coordination number attains zero when upper limit equals $r / d=1.0[9,8]$. Therefore, for the integral with such limit, the mean coordination number also goes to zero. Thus, to find physically reasonable estimate of these numbers and overcome the cited problem it is required to choose different upper limit of the integral. Two cases were considered following suggestions found in the literature. In the first case the upper limit of the integral was assumed to be $\lambda=1.5[7,11,14]$ and in the second case it was made equal to the distance corresponding to the location of the first minimum of the pair distribution function $[9,2]$.

The numerical procedure for calculation PDF function follows the one suggested in literature $[20,4]$. Around every cylindrical particle, a set of bins with the thickness $\Delta r$ are created and particles inside each bin are counted. With $N_{k}$ being the number of particles in $k$ th bin. After that the histogram of $N_{k}$ values is sorted in increasing radius $r$. In this way, the pair distribution function is obtained and given by the formula

$$
g_{2}(r)=\frac{N_{k} R^{2}}{2 N^{2} r \Delta r},
$$

where

$$
\Delta r=r_{k}-r_{k-1}
$$

is the width of the bin. The quantity $N$ in Eq. (3) corresponds to total number of particles in composite. The discrete radial distance $r$ is assumed to be in midway of every shell bin. Some bin width $\Delta r$ values were tested and the value of $0.0125 d$ was selected as adequate in these calculations. Pair distribution function, $g_{2}(r)$, was calculated for every single realization of geometry and at the end the ensemble averaged value of that function was calculated [4]. For numerical simulation an integral in Eq. (2) was approximated by a simple summation

$$
Z_{P D F}=\sum_{r=1}^{r=r_{\min }} 2 \pi r g_{2}(r) \frac{N}{A} \Delta r .
$$

The correlation function in range $0<r / d r<1$ is zero so the lower integration limit was set at $r=1.0$. The upper limit was the first minimum 
of the pair distribution function, $X_{0}=r_{\min } / d$, or the value of $r=1.5 d$, respectively. In order to avoid the numerical determination of the first minimum a linear correlation between location of the first minimum of the $\mathrm{PDF}$ function and volume fraction of particles was found basing on data obtained for the ensemble averaging:

$$
X_{0}=-1.0171 f+2.2597,
$$

where $f$ is the volume fraction. The values $X_{0}$ calculated from the correlation above were used as the approximate first minimum of the neighbour particle locations. For $f=0.49,0.4$, and 0.3 this correlation predicts $X_{0}=1.7613,1.8529$, and 2.0321 , respectively. It was observed that locations of the first minima are not strongly sensitive to the size of particle so that the created correlation depends only on volume fraction (Figs. 4-6).

The PDF method is generally applicable to the large groups of particles due to its statistical nature or according to the ergodic hypothesis to smaller groups of particles but for large number of realizations [21]. The research reported in the literature usually concerns very huge (infinite) media and it is the common practice to simulate only small representative parts of them with special procedures (i.e., periodic boundaries) to omit direct influence of boundaries on the obtained results. Typically, in calculations of PDF one particle is placed in the centre of system and PDF (RDF) is calculated as ensemble average for that particle for huge amount of realizations [4]. On the contrary, in this paper it was assumed that investigated system is the whole composite with boundary effects and no central particle present.

\subsection{Sequential analysis method}

The sequential analysis (SA) is a different and simpler way to find the coordination number. The basis of that method is the counting process of particles in the nearest neighbourhood of the analysed particle. The mean coordination number, (Eq. (2)), is obtained by summing CNs of every particle and dividing this sum by number of particles:

$$
Z_{S A}=\frac{\sum_{i=1}^{Z} Z_{C_{i}}}{N}
$$

In the literature, this method was introduced for sets of self-touching particles [3]. Here we propose to use the SA method for nontouching (in general) sets of parallel fibres and introduce the concept of neighbourhood with the 
same radius as for PDF method.

It was assumed that the neighbourhood area is contained inside a circle located in the centre of every cylindrical particle and has the radius $r / d=1.5$ or $r / d=X_{0}$ found from Eq. (6). If any other particle centre is located inside this circle, it is counted as a neighbour. In calculations also the cumulative coordination number, (Eq. (1)), for the SA method was determined according to the formula

$$
Z_{S A}(r)=\frac{\sum_{i=1}^{N} Z_{C_{i}}(r)}{N} .
$$

\subsection{Approaches to $\mathrm{CN}$ determination}

Four different mean coordination numbers were calculated: $Z_{1}, Z_{2}, Z_{3}$, and $Z_{4}$. The first two were calculated from the first minima of the PDF functions given by Eq. (6). Coordination number $Z_{1}$ was calculated by direct integration of pair distribution functions and $Z_{2}$ was determined by the sequential analysis. The coordination numbers $Z_{3}$ and $Z_{4}$ were calculated for neighbourhood area corresponding to the radius equal to $r / d=1.5$ either by the PDF integration or by the SA, respectively.

\section{Results and discussion}

\subsection{Comparison of PDF and RDF functions}

The resulting ensemble PDF functions were compared with the available numerical results obtained by Monte Carlo method [22], theoretical calculations carried by Percus-Yevick approximation [22] and the recent residual Helmholtz energy calculations introduced by Boublik [15]. Our numerical data for finite systems were matched with the RDF functions for the infinite systems corresponding to the particle volume fractions $f=0.3628,0.4535$, and 0.5441. The last volume fraction $(f=0.49)$ was different and chosen because of difficulty to obtain the ensemble averaged results in a reasonable computational time for the case $f=0.5441$ close to jamming limit (it is about $f=0.5470[8])$.

The results reveal that the PDFs obtained are close to RDFs for the region below the first minimum (Figs. 1 and 2). Nevertheless, above this point effects of the external boundary appear and the PDFs slowly converge to zero. Therefore, all coordination numbers were calculated using the region under the first minimum so that the influence of boundary effects 


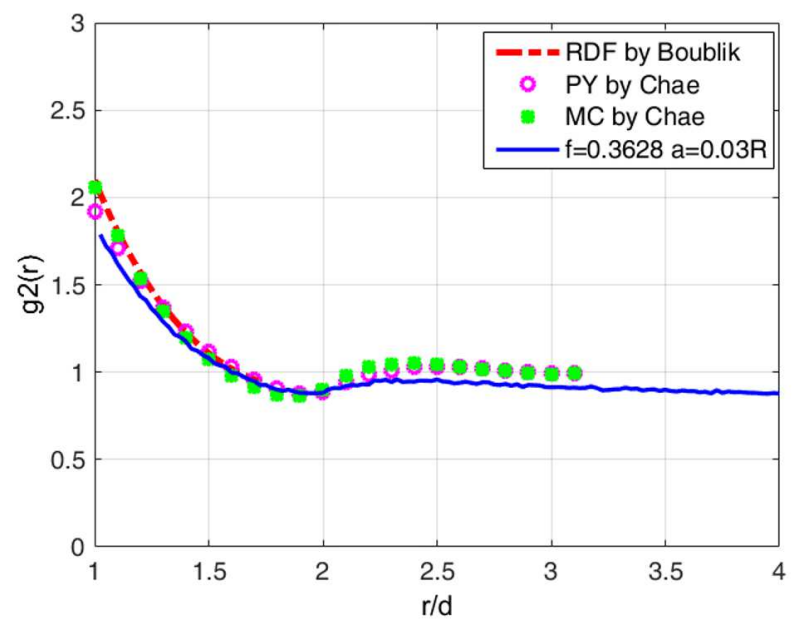

Figure 1: Comparison of numerical ensemble averaged PDF's for $f=0.3628$ with radius $a=0.03 R$ ( $R$ is a matrix radius) with data obtained from Monte Carlo (MC) simulations of RDF's, obtained from Percus-Yevick (PY) approximation [22] and based on the residual Helmholtz energy calculations [15].

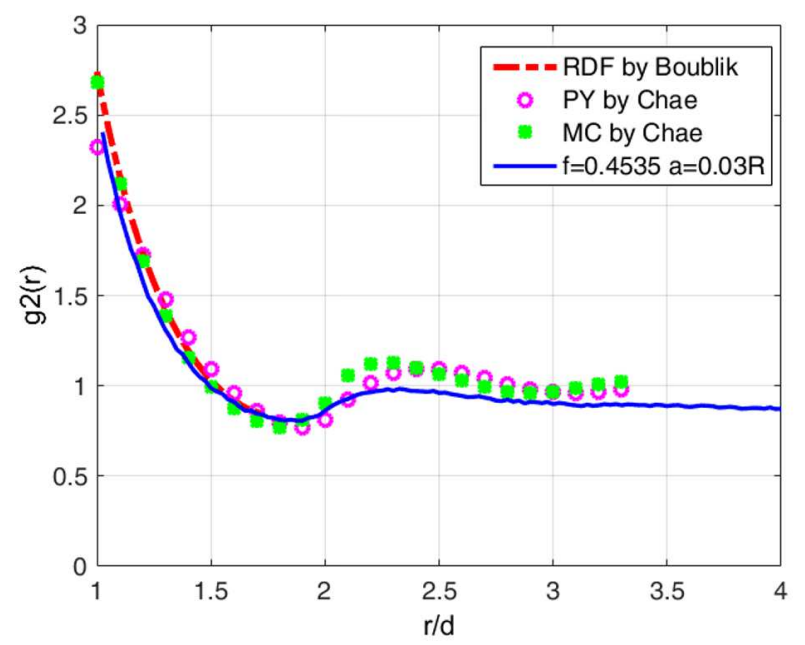

Figure 2: Comparison of numerical ensemble averaged PDF's for $f=0.4535$ with radius $a=0.03 R$ with data obtained from Monte Carlo (MC) simulations of RDF's [22], obtained from Percus-Yevick (PY) approximation [22] and based on the residual Helmholtz energy calculations [15]. 


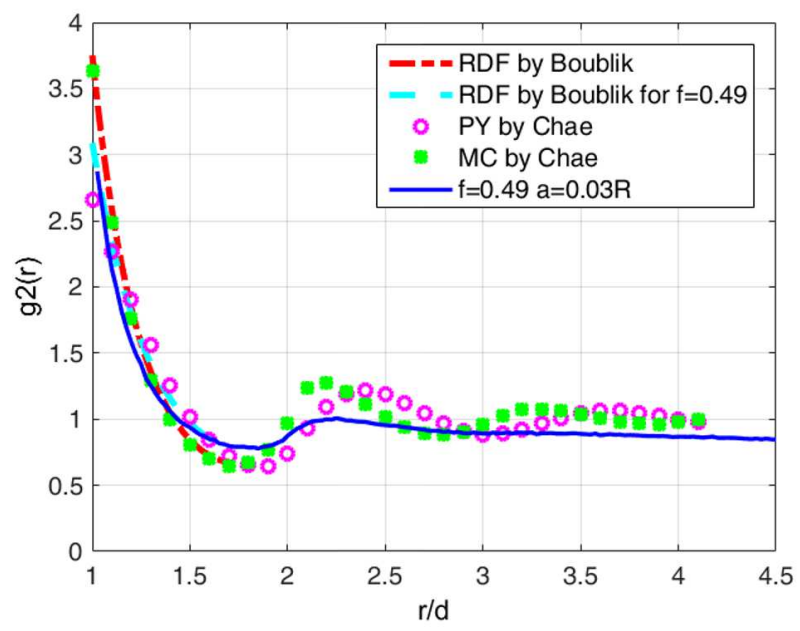

Figure 3: Comparison of numerical ensemble averaged PDF's for volume fraction $f=$ 0.49 and radius $a=0.03 R$ with data obtained for $f=0.5441$ from Monte Carlo (MC) simulations [22] of RDF's, obtained from Percus-Yevick (PY) approximation [22] and based on the residual Helmholtz energy calculations [15].

is insignificant. For $f=0.49$ case an acceptable agreement between PDF and RDF based on Helmholtz energy calculations was observed (Fig. 3). It seems that this simple method gives proper RDF for $r / d<\sqrt{3}$. It was possible to calculate $g_{2}(r)$ above this point but the results were found less satisfactory [15].

\subsection{Ensemble averaged results}

The mean coordination numbers obtained both from the PDF and SA approaches were evaluated for three volume fractions of particles $f=$ $0.3,0.4,0.49$, and for four different fibres diameters $a / R=0.03,0.04,0.05$, and 0.06. All CNs were averaged over one thousand realizations. The resulting PDFs reveal that all four density functions converge asymptotically to zero (Figs. 4-6). The respective functions with same volume fraction and different particle size are generally similar in shape. The smaller cylindrical particle diameter gives higher amount of particles spread in the same volume of the matrix. Hence, in the limit case of infinitely small particles, the PDF function will gain shape of radial distribution function converging to one. For lower values of the dimensionless distance $\left(r / d<X_{0}\right)$ functions for 
the same volume fractions overlap each other. Above this limit decreasing slope with decreasing size of particles can be observed for PDF functions. As it was discussed before, region $\left(r / d<X_{0}\right)$ is important for calculation of the coordination number. Therefore, it seems correct to conclude that the external boundary of the domain exerts little influence on CNs due to overlapping.

Shifting of the first minimum locations of the PDFs was observed for the same size of particles and different volume fractions (Figs. 4-6). The location of the first minimum increases with decreasing volume fraction. For high volume fraction it is very close to $r / d=\sqrt{3}$ but for lower volume fraction it tends to $r / d=2.0$. Equation (6) takes this effect into account without the necessity of numerical search of the first minimum. For high volume fractions $(f=0.49)$ first minima are simple to observe (Fig. 4) but for lower fraction $(f=0.3)$ these minima are difficult to perceive (Fig. 6). Finally, for the volume fractions $f<0.3$ the minimum in the PDF disappears. This effect causes a problem to find the location of the minimum numerically and to calculate CNs using PDF method.

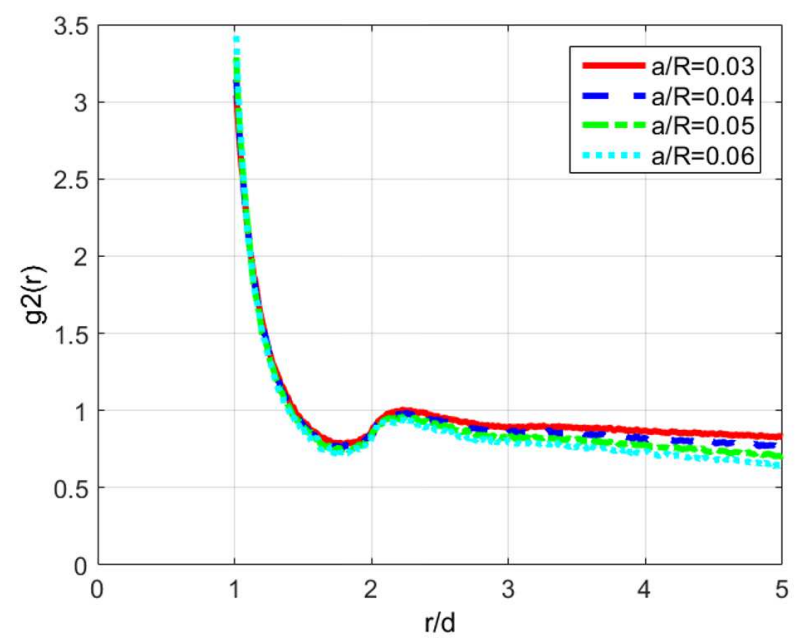

Figure 4: PDF's for volume fraction $f=0.49$ and different diameter of fibres.

The first thing which should be highlighted is the equivalence of results following from the sequential analysis and pair distribution function method for the same neighbourhood region (Tab. 1). The relative difference between $Z_{1}$ and $Z_{2}$ is less than $0.6 \%$ and for $Z_{3}$ and $Z_{4}$ it is less than $1.3 \%$. 


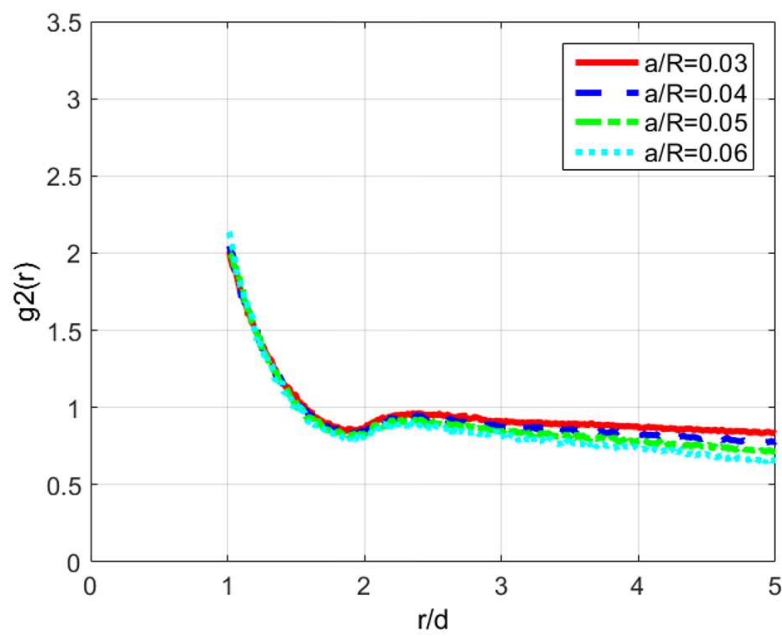

Figure 5: PDF's for $f=0.4$ and for different diameter of fibres.

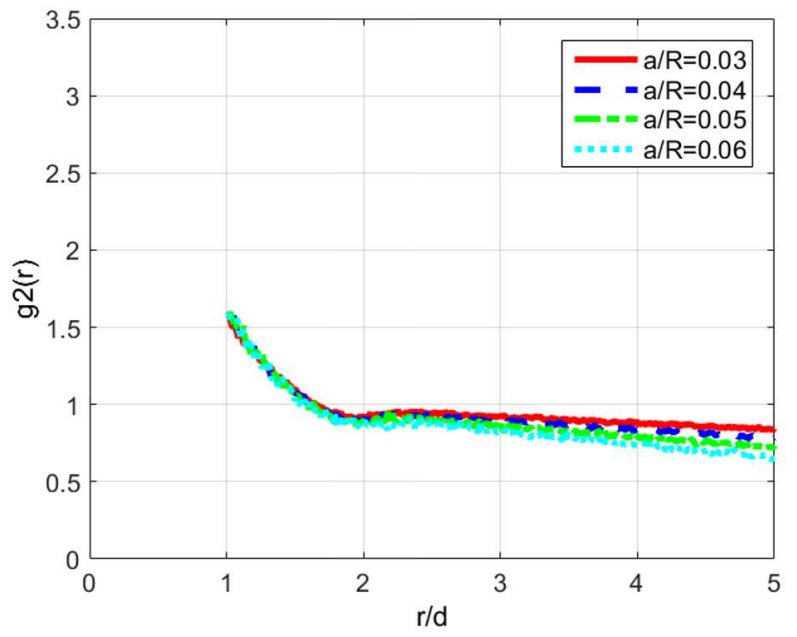

Figure 6: PDF's for $f=0.3$ for different sizes of diameter of fibres.

The CNs calculated from the first minimum have a tendency to be higher for smaller particles and the difference can be as high as 2-3\% between both cases when $a=0.03 R$ and $a=0.06 R$. For the CNs with $r / d=1.5$ such effect is observable only for $Z_{3}$ for $f=0.49$ and the difference is close to 
Table 1: CNs calculated from the ensemble averaged PDF and SA methods. Integration limit equal to first minima of $\mathrm{PDF}$ function was applied to $Z_{1}$ (PDF) and $Z_{2}$ (SA). Constant integration limit $r / d=1.5$ was applied to $Z_{3}(\mathrm{PDF})$ and $Z_{4}$ (SA); $\sigma$ is standard deviation of the $\mathrm{CN}$.

\begin{tabular}{ccccccccccc}
\hline $\mathbf{a} / \mathbf{R}$ & $\mathbf{f}$ & $\mathbf{N}$ & $\mathbf{Z 1}$ & $\sigma$ & $\mathbf{Z 2}$ & $\sigma$ & $\mathbf{Z 3}$ & $\sigma$ & $\mathbf{Z 4}$ & $\sigma$ \\
\hline $\mathbf{0 . 0 3}$ & $\mathbf{0 . 4 9}$ & $\mathbf{5 4 4}$ & $\mathbf{5 . 1 2 7 4}$ & 0.0465 & $\mathbf{5 . 1 5 5 3}$ & 0.0466 & $\mathbf{3 . 7 2 2 8}$ & 0.0457 & $\mathbf{3 . 7 5 7 1}$ & 0.0454 \\
& $\mathbf{0 . 4}$ & $\mathbf{4 4 4}$ & $\mathbf{4 . 6 2 4 1}$ & 0.0539 & $\mathbf{4 . 6 0 6 8}$ & 0.0532 & $\mathbf{2 . 8 1 5 3}$ & 0.0502 & $\mathbf{2 . 8 4 7 4}$ & 0.0504 \\
& $\mathbf{0 . 3}$ & $\mathbf{3 3 3}$ & $\mathbf{3 . 7 7 4 0}$ & 0.0690 & $\mathbf{3 . 7 6 7 3}$ & 0.0691 & $\mathbf{1 . 9 0 8 5}$ & 0.0610 & 1.9332 & 0.0622 \\
& & & & & & & & & & \\
0.04 & $\mathbf{0 . 4 9}$ & $\mathbf{3 0 6}$ & $\mathbf{5 . 0 7 9 8}$ & 0.0605 & $\mathbf{5 . 1 0 6 4}$ & 0.0616 & $\mathbf{3 . 7 7 4 2}$ & 0.0632 & $\mathbf{3 . 7 4 0 9}$ & 0.0641 \\
& $\mathbf{0 . 4}$ & $\mathbf{2 5 0}$ & $\mathbf{4 . 5 8 8 6}$ & 0.0719 & $\mathbf{4 . 5 7 1 4}$ & 0.0720 & $\mathbf{2 . 8 7 6 6}$ & 0.0695 & $\mathbf{2 . 8 4 6 0}$ & 0.0687 \\
& $\mathbf{0 . 3}$ & 187 & $\mathbf{3 . 7 5 4 4}$ & 0.0901 & $\mathbf{3 . 7 4 7 3}$ & 0.0896 & $\mathbf{1 . 9 5 8 1}$ & 0.0840 & 1.9337 & 0.0843 \\
& & & & & & & & & & \\
0.05 & $\mathbf{0 . 4 9}$ & 196 & $\mathbf{5 . 0 3 1 2}$ & 0.0777 & $\mathbf{5 . 0 5 7 9}$ & 0.0776 & $\mathbf{3 . 7 5 1 2}$ & 0.0743 & $\mathbf{3 . 7 1 8 0}$ & 0.0747 \\
& $\mathbf{0 . 4}$ & $\mathbf{1 6 0}$ & $\mathbf{4 . 5 5 7 9}$ & 0.0914 & $\mathbf{4 . 5 4 1 8}$ & 0.0914 & $\mathbf{2 . 8 7 8 3}$ & 0.0917 & $\mathbf{2 . 8 4 7 2}$ & 0.0915 \\
& $\mathbf{0 . 3}$ & $\mathbf{1 2 0}$ & $\mathbf{3 . 7 2 4 5}$ & 0.1182 & $\mathbf{3 . 7 1 7 4}$ & 0.1180 & $\mathbf{1 . 9 6 3 5}$ & 0.1047 & 1.9400 & 0.1050 \\
& & & & & & & & & & \\
0.06 & $\mathbf{0 . 4 9}$ & 136 & $\mathbf{4 . 9 7 7 6}$ & 0.0919 & $\mathbf{5 . 0 0 3 4}$ & 0.0920 & $\mathbf{3 . 6 6 3 9}$ & 0.0946 & $\mathbf{3 . 6 9 6 6}$ & 0.0946 \\
& $\mathbf{0 . 4}$ & $\mathbf{1 1 1}$ & $\mathbf{4 . 5 1 8 8}$ & 0.1104 & $\mathbf{4 . 5 0 2 6}$ & 0.1100 & $\mathbf{2 . 8 0 5 0}$ & 0.1038 & $\mathbf{2 . 8 3 4 8}$ & 0.1041 \\
& $\mathbf{0 . 3}$ & $\mathbf{8 3}$ & $\mathbf{3 . 6 9 9 4}$ & 0.1432 & $\mathbf{3 . 6 9 2 3}$ & 0.1431 & $\mathbf{1 . 9 0 7 3}$ & $\mathbf{0 . 1 3 2 8}$ & $\mathbf{1 . 9 2 9 9}$ & 0.1330 \\
\hline \hline
\end{tabular}

1.6\%. In general the standard deviations for the analysed cases (Tab. 1) increase with increasing fibres radius due to the smaller number of fibres and poorer statistics.

More general quantity, the cumulative coordination number was also investigated and convergence between the SA and PDF methods was observed for a wide domain of possible neighbourhood radii (Fig. 7). This result shows again that the methods are equivalent and the simple SA method can be used instead of PDF in two dimensional systems. Dependence of the cumulative coordination number on the radius of fibres is presented in Fig. 8. The cumulative coordination number increases with decreasing radius of fibres for the same volume fraction and difference between top and bottom value becomes higher with increasing integration limit $r / d$. It is possible to observe that near to the contact region $Z(r)$ is concave for high volume fraction. The same effect was observed by Stillinger [8] otherwise for higher $r / d$ opposite behaviour is visible. Upper single line on Fig. 8 shows expected behaviour of $Z(r)$ near the jamming limit $(f=0.54689)$ [8]. The shape of the cumulative coordination number for $r / d>X_{0}$ predicted in 


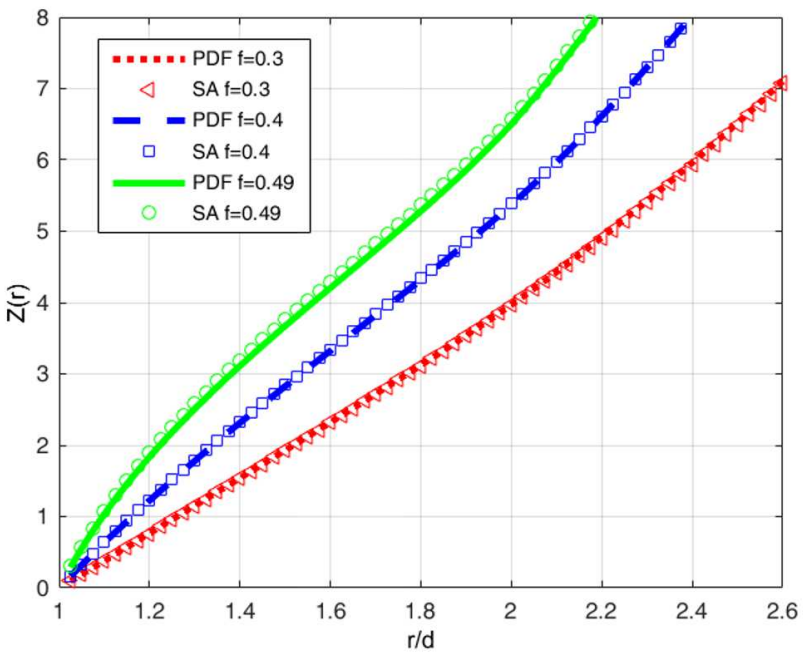

Figure 7: Cumulative coordination numbers for $a=0.03 R$ versus dimensionless distance (neighbourhood radius) and volume fraction.

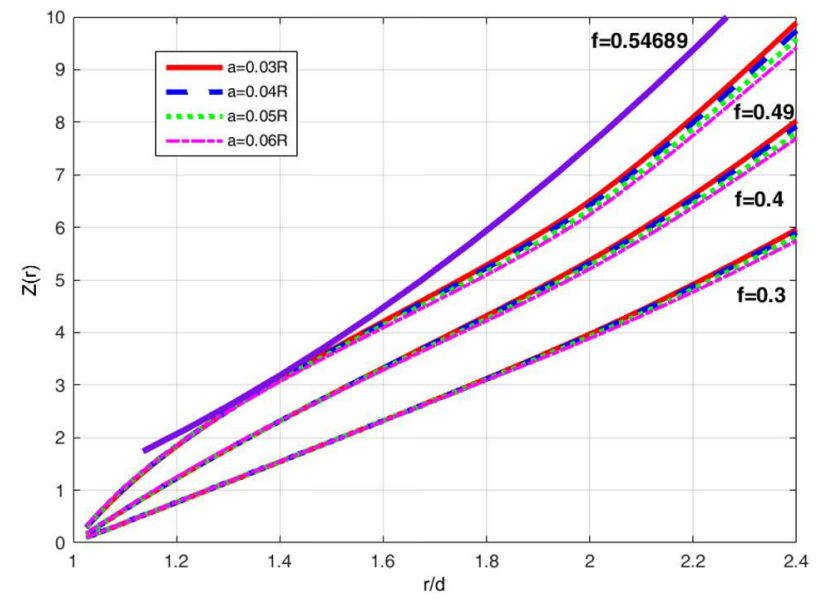

Figure 8: Cumulative coordination number for the PDF method calculated for different particle radii and different volume fractions compared with approximated relation for the cumulative CN proposed by Stillinger $(f=0.54689)$ near the jamming limit (upper thick line) [8]. 
this paper are slightly different than Stillinger's predictions where the main reason of discrepancy is convergence of the PDF function to zero (Fig. 8). In consequence, the integral $Z(r)$ will be affected by this convergence and therefore it should be smaller than for the RDF case presented in [8].

\subsection{Single geometry results}

It is well known that the sequential analysis is applicable for any amount of particles but it is not the case for the PDF method. Because of its statistical nature the PDF function needs sufficient amount of data to be constructed. Comparison of coordination numbers obtained by SA and PDFs is presented here to outline the limit for PDF method.

Six different single geometry realizations were analysed for two extreme particle diameters $a=0.03 R$ and $a=0.06 R$. Figures 9-14 compare PDFs fitted by spline with the theoretical prediction of RDF given by Boublik [15] with the ensemble averaged data for one thousand realizations. Obtained spline fits of the PDFs for single geometry show their unexpected compatibility with ensemble averaged data and theoretical predictions (Figs. 9-14).

Two problems appear during spline calculations - the first minimum location changes its value significantly in comparison to its actual position and shape of PDF near the contact is deformed. Therefore calculation of $\mathrm{CN}$ by integration will lead to difference with ensemble data calculations. Additionally, oscillations of spline appear for $r / d>X_{0}$ but they can be reduced by choosing a proper spline smoothing parameter and some further research is here necessary [17]. Generally speaking the shape of fitted spline can be improved by changing the smoothing parameter and in consequence the problem with poor spline fitting near contact region and problem with spline first minimum location could be solved. Nevertheless using the proposed approximate formula given by Eq. (6) the problem of proper estimation of location of the PDF minimum can be omitted. Concluding, the shape of the PDFs obtained in numerical simulations with one realization are quite correct but resulting coordination numbers can be treated only as unreliable approximation.

What is interesting, the coordination numbers obtained for those randomly chosen geometries are consistent with results from the ensemble average (Tabs. 1, and 2). An attempt was undertaken to integrate fitted splines but the results were found not as satisfactory as from the direct integration of resulting PDF data. For $a=0.03 R$ the relative difference between $\mathrm{CNs}$ is less than $2 \%$ for all cases. When $a=0.06 R$ this relative dif- 


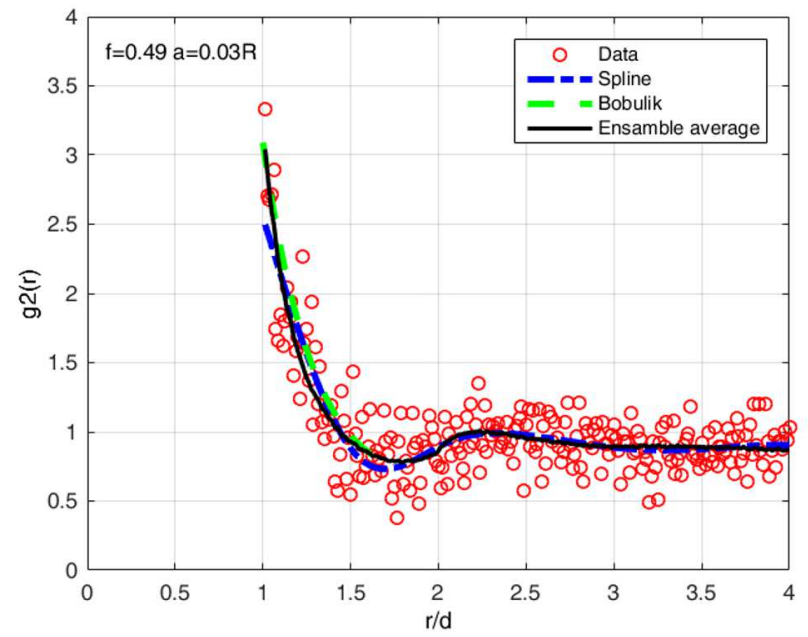

Figure 9: One realization of the PDF for the volume fraction $f=0.49$ and the fibre radius $a=0.03 R$ with the number of particles equals $N=544$.

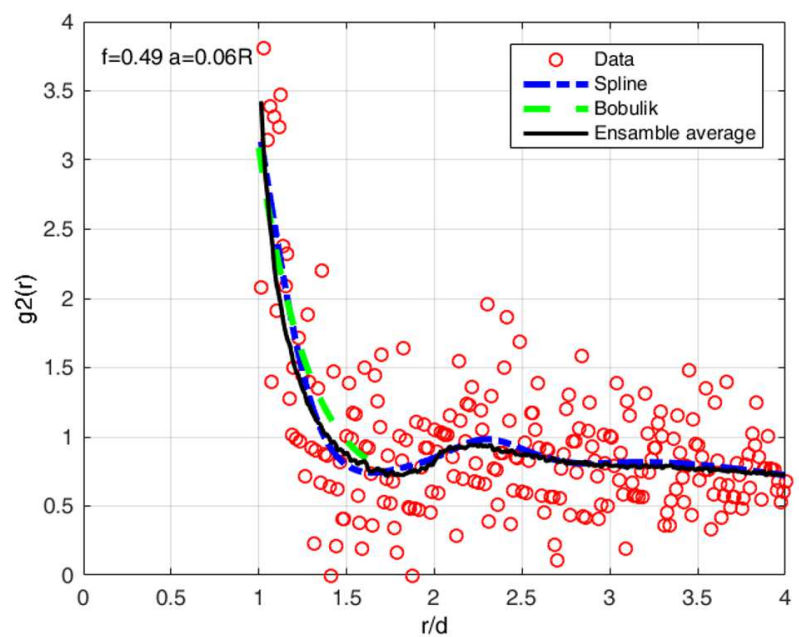

Figure 10: One realization of the PDF for the volume fraction $f=0.49$ and fibre radius $a=0.06 R$ with $N=136$.

ference is less than $8 \%$. Comparing $Z_{1}$ and $Z_{3}$ calculated by the PDF with $Z_{2}$ and $Z_{4}$ calculated by the SA differences are estimated to lie between $0.1-1.3 \%$ - see Tab. 2 . 


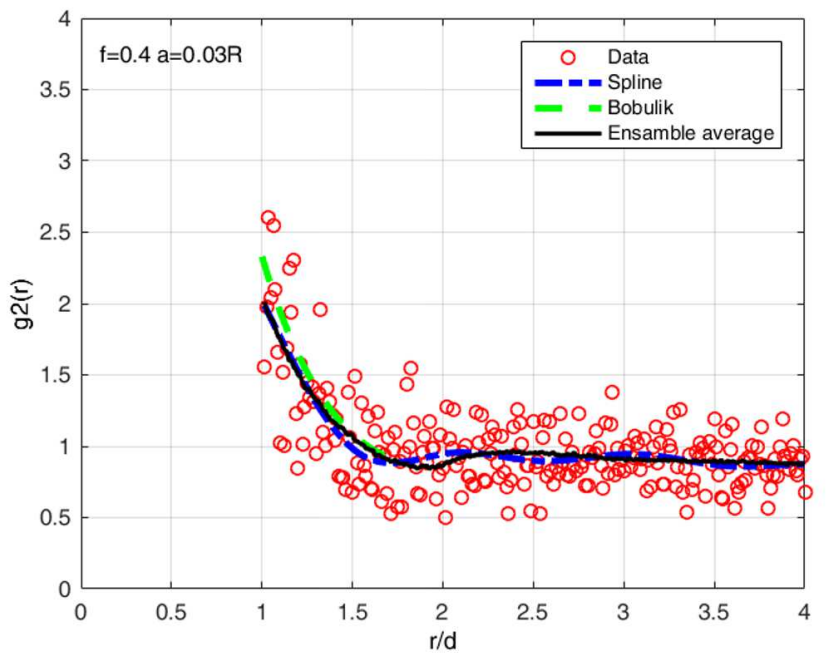

Figure 11: One realization of the PDF for the volume fraction $f=0.4$ and the fibre radius $a=0.03 R$ with $N=444$.

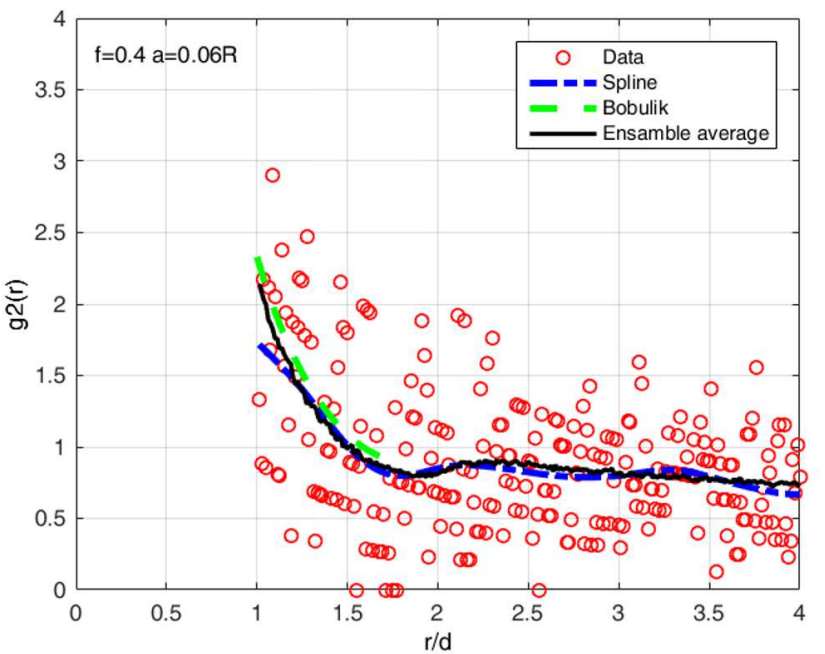

Figure 12: One realization $\mathrm{PDF}$ with volume fraction $f=0.4$ and fibre radius $a=0.06 R$ with $N=111$.

\subsection{Correlation between volume fraction and coordination number}

A lot of correlations between the coordination number and the volume fraction were proposed in literature for heterogeneous media made of spherical 


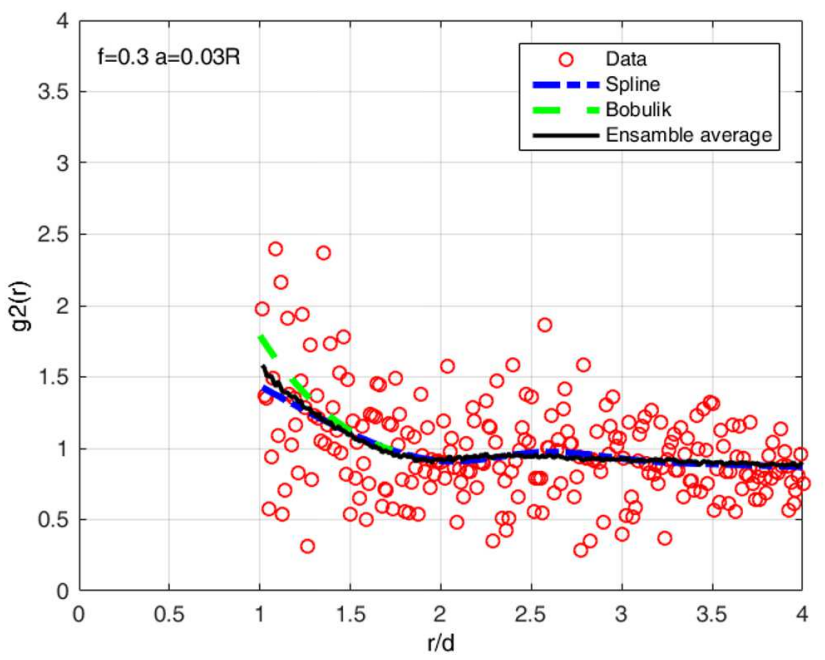

Figure 13: One realization PDF with volume fraction $f=0.3$ and fibre radius $a=0.03 R$ with $N=333$.

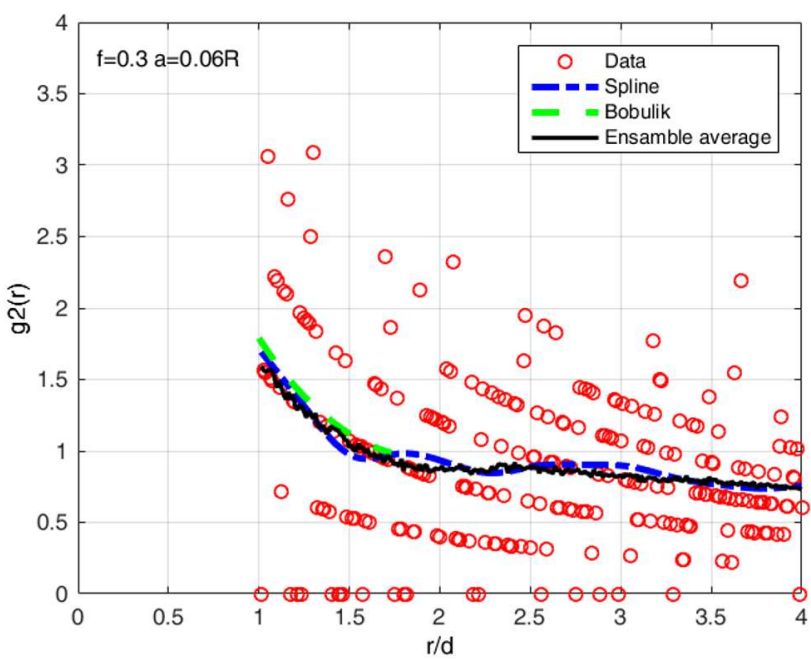

Figure 14: One realization PDF with volume fraction $f=0.3$ and fibre radius $a=0.06 R$ with $N=83$

particles dispersed in a matrix $[11,5,12]$. For random sequential addition with $\lambda=1.5$ and cylinders the numerical data for $\mathrm{CN}$ can be found in 
Table 2: Examples of coordination numbers for one geometry realization.

\begin{tabular}{ccccccc}
\hline \hline $\mathbf{f}$ & $\mathrm{a} / \mathrm{R}$ & $\mathrm{N}$ & $\mathrm{Z1}$ & $\mathrm{Z2}$ & $\mathrm{Z3}$ & $\mathrm{Z4}$ \\
\hline 0.49 & 0.03 & 544 & 5.1213 & 5.1397 & 3.6949 & 3.7096 \\
0.4 & 0.03 & 444 & 4.6216 & 4.5901 & 2.7748 & 2.7883 \\
0.3 & 0.03 & 333 & 3.7297 & 3.7237 & 1.9219 & 1.9399 \\
& & & & & & \\
0.49 & 0.06 & 136 & 5.1471 & 5.1618 & 3.6176 & 3.6618 \\
0.4 & 0.06 & 111 & 4.2523 & 4.2342 & 2.7387 & 2.7748 \\
0.3 & 0.06 & 83 & 3.9759 & 3.9518 & 1.9277 & 1.9518 \\
\hline \hline
\end{tabular}

Danawabichakul paper [14] and the proper fitting with the second order polynomial to these data (the lower line on Fig. 15) allowed us to present the following correlation:

$$
Z(f)=6.8898 f^{2}+4.3608 f .
$$

Our numerical data (Tab. 1) agrees very well with this correlation in spite of the finite system considered in calculations. For $Z_{3}$ relative differences for $a / R=0.03,0.04$, and 0.05 lie between $0.4-1.8 \%$ while for $a=0.06 R$ they are between $1.9-3.7 \%$. In the case of $Z_{4}$ all results fit even better and they are between $0.02-2.5 \%$. Value $Z=4.482605$ is the mean coordination number near jamming limit obtained by Stillinger [8] for volume fraction $f=0.544689$. It matches Eq. (9) with relative difference close to $1.47 \%$ (Fig. 15). Presented results indicate that the obtained correlation and our numerical data are consistent with the literature results.

The second correlation was obtained for coordination numbers calculated from the neighbourhood radii corresponding to the first minima $\left(Z_{1}\right.$ in Tab. 1) and fitted by logarithmic curve (the upper line on Fig. 15):

$$
Z(f)=2.6967 \ln (f)+7.002 \text {. }
$$

Unfortunately no data were found in literature to confirm this result. It could only be expected that this curve should converge to the highest possible coordination number equal to 6 for the most dense packing of fibres corresponding to $f=0.9068$ for the hexagonal structure [8], but Eq. (10) unfortunately does not meet this requirement. 


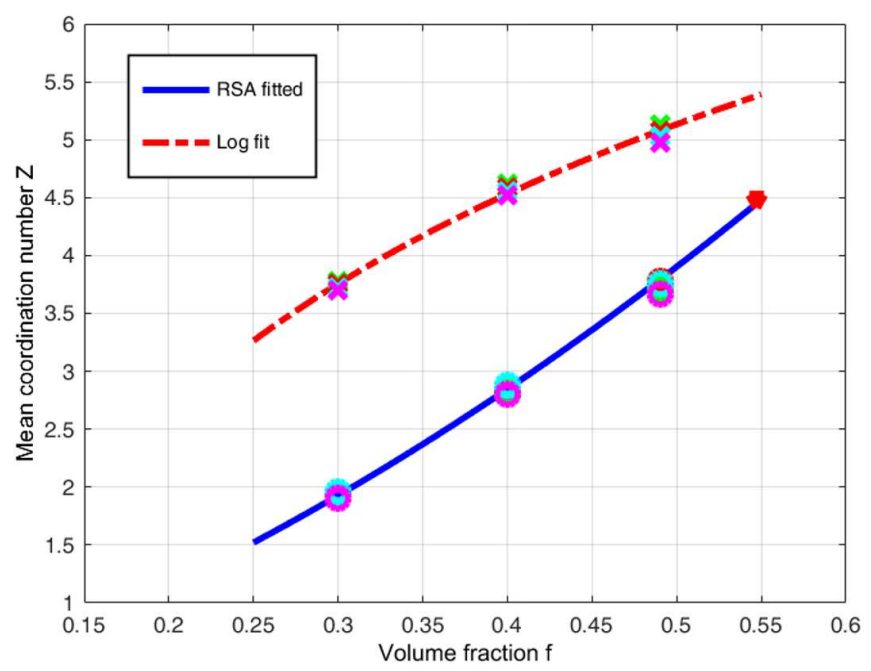

Figure 15: Correlations for dependence of mean coordination number on volume fraction. Lower line presents polynomial fit of numerical data for RSA packing scheme [14] for integration limit $r / d=1.5$ compared with $Z_{1}$ values for $a=0.03 R, a=$ $0.04 R, a=0.05 R$, and $a=0.06 R$ presented by circles. Upper line is a logarithmic fit of $Z_{3}$ numerical data (presented by crosses) for integration of PDF with $X_{0}$ as upper integration limit. Triangle on lower line presents mean coordination number of RSA for jamming limit obtained by Torquato [8].

\section{Conclusions}

Results of calculations of the cumulative coordination numbers show that the Sequential Analysis and Pair-Distribution Function integration are equivalent for wide range of neighbourhood radii (Fig. 7 and Tab. 1). This indicates that instead of conducting complicated integration by the PDF method it is possible to perform simple counting and summation using the SA method. Moreover, the modified SA method presented here should be more general and applicable for any number of particles (even very small) without concern about statistics of the medium structure. Nevertheless, for the investigated single realization examples (Tab. 2) the CNs obtained from the PDF method were surprisingly consistent with results given by the SA method, despite of complete loss of pair distribution function shape (Figs. 9-14). This allows to calculate the CN from the single geometry pattern by the PDF method but such operation should be performed with high caution. 
For fixed integration limits of the cumulative coordination number: $\lambda=1.5$ and $\lambda=X_{0}$, Eq. (2) gives mean coordination numbers and they are correlated with volume fraction (Fig. 15). The correlation between mean coordination number for $\lambda=1.5$ and volume fraction obtained for the RSA packing scheme, Eq. (9), is in good agreement with the literature data and such correlation could be helpful in engineering applications. One more correlation between volume fraction and coordination number calculated with integration limit equal to the first minimum of $\operatorname{PDF}\left(\lambda=X_{0}\right)$ was proposed, Eq. (10). The important conclusion derived from these two correlations is an insignificant influence of particle size due to the overlapping of PDF functions in neighbourhood region (Figs. 4-6). Additionally, it was shown that boundary effect of the PDF function should have small influence on determination of the coordination number (Figs. 1-3).

Concluding, the methods described in this paper would be useful to calculate coordination numbers of single geometries. This in turn will allow to correlate effective properties of fibre-reinforced composites with their microstructure.

Acknowledgements This work has been supported by the Polish National Centre for Research and Development within European Regional Development Fund under the Operational Program Innovative Economy No POIG.01.01.02-00-097/09 'TERMET - New structural materials with enhanced thermal conductivity'.

Received 21 March 2016

\section{References}

[1] Muller P.: Glossary of terms used in physical organic chemistry. Pure Appl. Chem. 66(1994), 5, 1077-1184.

[2] Vahvaselka K., Magnus J.M.: X-ray diffraction study of liquid sulfur. Phys. Scripta 38(1988), 5, 737-741.

[3] Jounnnot-Chesney P., Jernot J. P., Lantuejoul C.: Practical determination of the coordination number in granular media. Image Anal. Stereol. 25(2006), 1, $55-61$.

[4] Torquato S.: Random Heterogeneous Materials - Microstructure and Macroscopic Proporties. Springer-Verlag, Berlin 2002.

[5] Antwerpen W., Rousseau P.: A review of correlations to model the packing structure and effective thermal conductivity in packed beds of mono-sized spherical particles. Nuclear Eng. Design 240(2010), 7, 1803-1818. 
[6] Furmański P., Seredyński M., Łapka P., Banaszek J.: Micro-macro model for prediction of local temperature distribution in heterogenous and two-phase media. Arch. Thermodyn. 35(2014), 3, 81-103.

[7] Nayak A. L., Tien C.: A statistical thermodynamic theory for coordination-number distribution and effective thermal conductivity of random packed beds. Int. J. Heat Mass Trans. 21(1978), 6, 669-676.

[8] Largo J., Solana J.: Theory and computer simulation of the coordination number of square-well fluids of variable width. Fluid Phase Equilibr. 193(2002), 1-2, 277287.

[9] Torquato S., Uche O.U., Stillinger F.H.: Random sequential addition of hard spheres in high euclidean dimensions. Phys. Rev. E 74(2006),6, 061308-061308-10.

[10] Huerta A., Naumis G.G.: Role of rigidity in the fluid-solid transition. Phys. Rev. Lett. 90(2003), 145701-1-145701-4.

[11] Suzuki M., Oshima T.: Estimation of the co-ordination number in a multicomponent mixture of spheres. Powder Technol. 35(1983), 2, 159-166.

[12] Tassopoulos M., Rosner D.: Microstructural descriptors characterizing granular deposits. AIChEJ. 38(1992), 1, 15-25.

[13] ZARICHNYAK Y.P.: Generalization of data on the dependence of the coordination number on the porosity in the fillings of sintered or pressed granular materials. Inzhenerno-Fizicheskii Zhurnal 39(1980), 5, 862-865 (in Russian).

[14] Guo X., Riebel U.: Theoretical direct correlation function for two-dimensional fluids of monodisperse hard spheres. J. Chem. Phys. 125(2006), 14, 144504.

[15] Danwanichakul P., Glandt E.D.: Continutiy between disorder and order in the sequential deposition of particles. Chem. Eng. Commun. 192(2005), 11, 1405-1423.

[16] Boublik T.: Structure of two-dimensional hard disk systems. Simple geometric method. Fluid Phase Equilibr. 316(2012), 1-5.

[17] Cadilhe A., Araujo N., Privman V.: Random sequential adsorption: From continuum to lattice and pre-patterned substrates. J. Phys. Cond. Mat. 19(2007), 6, 065124.

[18] Bochkanov S., Bystritsk V.: ALGLIB User Guide. http://www.alglib.net, 2012.

[19] Hopkins A.B., Stillinger F., Torquato S.: Dense sphere packings from optimized correlation functions. Phys. Rev. E 79(2009), 3, 031123-1-031123-10.

[20] Furmański P.: Heat Conduction in Composites: Homogenization and Macroscopic Behavior. Appl. Mech. Rev. 50(1997), 6, 327-356.

[21] Crawford J., Torquato S., Stillinger F.: Aspects of correlation function realizability. J. Chem. Phys. 119(2003), 14.

[22] Huang K.: Introduction to Statistical Physics. PWN, Warszawa, 2006 (in Polish).

[23] Chat D. C., ReE T., REE F.H.: Radial distribution functions and equation of state of the hard-disk fluid. J. Chem. Phys. 50(1969), 4, 1581-1589.

[24] Smith P.A., Torquato S.: Computer simulation results for the two-point probability function of composite media. J. Comput. Phys. 76(1988), 1, 176-191.

[25] Uche O.U., Stillinger F.H., Torquato S.: On the realizability of pair correlation functions. Physicá A 360(2006), 1, 21-36. 
[26] Meyer S., Song C., Jin Y., Wang K., MaKse H.A.: Jamming in two-dimensional packings. Physica A 389(2010), 22, 5137-5144.

[27] Ueda T., Matsushima T., Yamada Y.: Micro structures of granular materials with various grain size distributions. Powder Technol. 217(2012), 533-539. 